\title{
Effect of Weight Function in Nonlinear Part on Global Solvability of Cauchy Problem for Semi-Linear Hyperbolic Equations
}

\author{
Akbar B. Aliev', Anar A. Kazimov ${ }^{2}$ \\ ${ }^{1}$ Institute of Mathematics and Mechanics of NAS of Azerbaijan, Baku, Azerbaijan \\ ${ }^{2}$ Nakhchivan State University, Nakhchivan, Azerbaijan \\ Email: alievakbar@math.ab.az, anarkazimov1979@gmail.com
}

Received December 13, 2012; revised January 19, 2013; accepted January 30, 2013

\begin{abstract}
In this paper, we investigate the effect of weight function in the nonlinear part on global solvability of the Cauchy problem for a class of semi-linear hyperbolic equations with damping.
\end{abstract}

Keywords: Cauchy Problem; Wave Equation; Global Solvability; Weight Function; Semi-Linear Hyperbolic Equation

\section{Introduction}

Consider the Cauchy problem for the semi-linear wave equation with damping

$$
\begin{aligned}
& u_{t t}-\Delta u+u_{t}=a(x)|u|^{p},(t, x) \in[0, \infty) \times R^{n}, \\
& u(0, x)=u_{0}(x), u_{t}(0, x)=u_{1}(x), \quad x \in R^{n},
\end{aligned}
$$

where $\Delta=\frac{\partial^{2}}{\partial x_{1}}+\cdots+\frac{\partial^{2}}{\partial x_{n}^{2}}, a(x) \in L_{q}\left(R^{n}\right), q>1$

In the case when $a(x)$ is independent of $x$, the existence and nonexistence of the global solutions was investigated in the papers [1-8]. The authors interests are focused on so called critical exponent $p_{c}(n)$, which is the number defined by the following property: if $p>p_{c}(n)$ then all small data solutions of corresponding Cauchy problem have a global solution, while $1<p \leq p_{c}(n)$ all solutions with data positive on blow up in finite time regardless of the smallness of the data.

In the present paper we investigate the effect of the weight function $a(x)$ on global solvability of Cauchy problems (1) and (2).

\section{Statement of Main Results}

We consider the Cauchy problem for a class of semilinear hyperbolic equation

$$
\begin{aligned}
& u_{t t}+(-1)^{l} \Delta^{l} u+u_{t}=f(t, x, u),(t, x) \in[0, \infty) \times R^{n}, \\
& u(0, x)=u_{0}(x), u_{t}(0, x)=u_{1}(x), x \in R^{n},
\end{aligned}
$$

where $l=1,2, \cdots$

Throughout this paper, we assume that the nonlinear term $f(t, x, u)$ satisfies the following conditions:

1) $f(t, x, u)$ and $f_{t}(t, x, u)=\frac{\partial(t, x, u)}{\partial t}$ are continuous functions in the domain $[0, \infty) \times R^{n+1}$.

2) $f(t, x, 0)=0$, and

$$
\begin{aligned}
& \left|f\left(t, x, u_{1}\right)-f\left(t, x, u_{2}\right)\right| \\
& \leq a(x)\left(\left|u_{1}\right|^{p-1}+\left|u_{2}\right|^{p-1}\right)\left|u_{1}-u_{2}\right|,
\end{aligned}
$$

where

$$
\begin{aligned}
& a(x) \in L_{q}\left(R^{n}\right), q>1, \\
& p \in\left(1+\frac{2 l}{n}-\frac{1}{q},+\infty\right) \quad \text { for } n \leq 2 l, \\
& p \in\left(2-\frac{1}{q}, \frac{n(q-2)}{q(n-2 l)}\right) \text { for } 2 l<n<\frac{4 l q}{q+1} .
\end{aligned}
$$

In the sequel, by $\|.\|_{q}$, we denote the usual $L_{q}(\Omega)$ norm. For simplicity of notation, in particular, we write $\|$.$\| instead of \|.\|_{2}$. The constants $C, c$ used throughout this paper are positive generic constants, which may be different in various occurrences.

Theorem 1. Suppose that the conditions (5)-(8) are satisfied. Then there exists a real number $\delta_{0}>0$ such that, if

$$
\begin{gathered}
\left(u_{0}, u_{1}\right) \in \bigcup_{\delta_{0}}=\left\{(\varphi, \psi): \varphi \in W_{2}^{l}\left(R^{n}\right) \cap L_{1}\left(R^{n}\right),\right. \\
\psi \in L_{2}\left(R^{n}\right) \cap L_{1}\left(R^{n}\right), \\
\left.\|\varphi\|_{W_{2}^{l}\left(R^{n}\right)}+\|\varphi\|_{L_{1}\left(R^{n}\right)}+\|\psi\|_{L_{2}\left(R^{n}\right)}+\|\psi\|_{L_{1}\left(R^{n}\right)}<\delta_{0}\right\}
\end{gathered}
$$


Then problem (3) and (4) admit a unique solution

$$
u(t, x) \in C\left([0, \infty) ; W_{2}^{l}\left(R^{n}\right)\right) \cap C^{1}\left([0, \infty) ; L_{2}\left(R^{n}\right)\right)
$$

satisfied the decay property

$$
\begin{aligned}
& \sum_{|\alpha|=r}\left\|D^{\alpha} u(t, \cdot)\right\| \leq c(d)(1+t)^{-\frac{n+2 r}{4 l}}, \\
& t \in[0, \infty), r=0,1, \cdots, l \\
& \left\|u_{t}(t, \cdot)\right\| \leq c(d)(1+t)^{-\eta}, t>0,
\end{aligned}
$$

where

$$
\eta=\min \left\{1+\frac{n}{4 l}, \frac{n(p-1)}{4 l}+\frac{n}{2 l q}\right\}, c(\cdot) \in C\left(R_{+}, R_{+}\right) .
$$

\section{Proof of Theorem 1}

It is well known that if

$$
\|u(t, \cdot)\|_{W_{2}^{l}\left(R^{n}\right)}+\left\|u_{t}(t, \cdot)\right\|_{L_{2}\left(R^{n}\right)} \leq c, \quad t \in\left[0, T_{\max }\right),
$$

then $T_{\max }=+\infty$, i.e. problem (3) and (4) have a global solution (see for example [9]).

Using the Fourier transformation, Plancherel theorem and the Hausdorff-Young inequality, for the solution $u(t, x)$ we have the following inequalities (see [1]):

$$
\begin{gathered}
\sum_{|\alpha|=l}\left\|D^{\alpha} u(t, \cdot)\right\|_{L_{2}\left(R^{3}\right)} \leq c(1+t)^{-\frac{n+2 l}{4 l}} E\left(u_{0}, u_{1}\right) \\
+c \int_{0}^{t}(1+t-\tau)^{-\frac{n+2 l}{4 l}} \Phi(\tau) \mathrm{d} \tau ; \\
\|u(t, \cdot)\|_{L_{2}\left(R^{3}\right)} \leq c(1+t)^{-\frac{n}{4 l}} E\left(u_{o}, u_{1}\right) \\
+c \int_{0}^{t}(1+t-\tau)^{-\frac{n}{4 l}} \Phi(\tau) \mathrm{d} \tau \\
\left\|u_{t}(t, \cdot)\right\|_{L_{2}\left(R^{3}\right)} \leq c(1+t)^{-\frac{n}{4 l}-1} E\left(u_{o}, u_{1}\right) \\
+c \int_{0}^{t}(1+t-\tau)^{-\frac{n}{4 l}-1} \Phi(\tau) \mathrm{d} \tau
\end{gathered}
$$

where,

$$
\begin{gathered}
E\left(u_{o}, u_{1}\right)=\left\|u_{0}\right\|_{L_{1}\left(R^{3}\right)}+\left\|u_{1}\right\|_{L_{1}\left(R^{3}\right)} \\
+\left\|u_{0}\right\|_{W_{2}^{l-k}\left(R^{3}\right)}+\left\|u_{1}\right\|_{L_{2}\left(R^{3}\right)} \\
\Phi(\tau)=\|f(\tau, x, u(\tau, x))\|_{L_{1}\left(R^{3}\right)} \\
+\|f(\tau, x, u(\tau, x))\|_{L_{2}\left(R^{3}\right)}
\end{gathered}
$$

On the other hand, by virtue of condition $2^{\circ}$

$$
\|f(t, x, u)\|_{L_{1}\left(R^{n}\right)} \leq c \int_{R^{n}} a(x)|u(x)|^{p} \mathrm{~d} x
$$

and

$$
\|f(t, x, u)\| \leq c \int_{R^{n}} a^{2}(x)|u(x)|^{2 p} \mathrm{~d} x .
$$

Using the Holder inequality, from (16) we have

$$
\|f(t, x, u)\|_{L_{1}\left(R^{n}\right)} \leq c\left(\int_{R^{n}} a^{q}(x) \mathrm{d} x\right)^{1 / q}\left(\int_{R^{n}}|u(x)|^{\frac{p q}{q-1}} \mathrm{~d} x\right)^{\frac{q-1}{q}} .
$$

By virtue of condition (7), (8) and the multiplicative inequality of Gagliardo-Nirenberg type, we have

$$
\begin{aligned}
& \|f(t, \cdot, u(\cdot))\|_{L_{1}\left(R^{3}\right)} \\
& \leq c\|a(\cdot)\|_{L_{q}\left(R^{n}\right)}^{q}\|u\|^{p(1-\theta)} \cdot\left(\sum_{|\alpha|=l}\left\|D^{\alpha} u\right\|\right)^{p \theta},
\end{aligned}
$$

where

$$
\theta=\frac{n}{l}\left(\frac{1}{2}-\frac{q-1}{p q}\right),(\text { see [10]). }
$$

Analogously from (17) we have

$$
\begin{aligned}
& \|f(t, \cdot, u(\cdot))\| \\
& \leq c\|a(\cdot)\|_{L_{q}\left(R^{n}\right)}^{2}\|u\|^{2 p\left(1-\theta^{\prime}\right)} \cdot\left(\sum_{|\alpha|=l}\left\|D^{\alpha} u\right\|\right)^{2 p \theta^{\prime}},
\end{aligned}
$$

where

$$
\theta^{\prime}=\frac{n}{2 l}\left(1-\frac{q-2}{p q}\right)
$$

From (12), (16) and (20) we have the following estimates

$$
\begin{aligned}
& \sum_{\alpha \mid=l}\left\|D^{\alpha} u(t, \cdot)\right\| \leq c(1+t)^{-\frac{n+2 l}{4 l}} E\left(u_{0}, u_{1}\right) \\
& +c \int_{0}^{t}(1+t-\tau)^{-\frac{n+2 l}{4 l}}\left[\|u(\tau, .)\|^{p(1-\theta)} \cdot\left(\sum_{|\alpha|=l}\left\|D^{\alpha} u(\tau, .)\right\|\right)^{p \theta},(22)\right. \\
& \left.+\|u(\tau, .)\|^{2 p\left(1-\theta^{\prime}\right)} \cdot\left(\sum_{|\alpha|=l}\left\|D^{\alpha} u(\tau, .)\right\|\right)^{2 p \theta^{\prime}}\right] \mathrm{d} \tau \\
& \|u(t, \cdot)\| \leq c(1+t)^{-\frac{n}{4 l}} E\left(u_{0}, u_{1}\right) \\
& +c \int_{0}^{t}(1+t-\tau)^{-\frac{n}{4 l}}\left[\|u(\tau, .)\|^{p(1-\theta)} \cdot\left(\sum_{|\alpha|=l}\left\|D^{\alpha} u(\tau, .)\right\|\right)^{p \theta} .(23)\right) \\
& \left.+\|u(\tau, .)\|^{2 p\left(1-\theta^{\prime}\right)} \cdot\left(\sum_{|\alpha|=l}\left\|D^{\alpha} u(\tau, .)\right\|\right)^{2 p \theta^{\prime}}\right] \mathrm{d} \tau
\end{aligned}
$$

It follows from (22) and (23) that 


$$
\begin{aligned}
& \quad G_{1}(t) \leq c d+c(1-t)^{\frac{n}{4 l}} \int_{0}^{t}(1+t-\tau)^{-\frac{n}{4 l}} \\
& \times\left[(1-\tau)^{-\gamma} G_{1}^{p(1-\theta)}(\tau) G_{2}^{p \theta}(\tau)\right. \\
& \left.+(1-\tau)^{-\gamma^{\prime}} G_{1}^{2 p\left(1-\theta^{\prime}\right)}(\tau) G_{2}^{2 p \theta^{\prime}}(\tau)\right] \mathrm{d} \tau ; \\
& G_{2}(t) \leq c d+c(1-t)^{\frac{n+2 l}{4 l}} \int_{0}^{t}(1+t-\tau)^{-\frac{n+2 l}{4 l}} \\
& \times\left[(1-\tau)^{-\gamma} G_{1}^{p(1-\theta)}(\tau) G_{2}^{p \theta}(\tau)\right) \\
& \left.+(1-\tau)^{-\gamma^{\prime}} G_{1}^{2 p\left(1-\theta^{\prime}\right)}(\tau) G_{2}^{2 p \theta^{\prime}}(\tau)\right] \mathrm{d} \tau
\end{aligned}
$$

where $G_{1}(t)$ and $G_{2}(t)$ are defined by

$$
\begin{aligned}
& G_{1}(t)=(1-t)^{\frac{n}{4 l}}\|u(t, \cdot)\|, \\
& G_{2}(t)=(1-t)^{\frac{n+2 l}{4 l}} \sum_{|\alpha|=l}\left\|D^{\alpha} u(t, \cdot)\right\|,
\end{aligned}
$$

and

$$
\gamma=\frac{n p}{4 l}+\frac{p \theta}{2}, \gamma^{\prime}=\frac{n p}{2 l}+p \theta^{\prime} .
$$

Then, we have from (19), (21) and (28) that

$$
\begin{aligned}
& \gamma=\frac{n p}{4 l}+\frac{p}{2} \cdot \frac{n}{l}\left(\frac{1}{2}-\frac{q-1}{p q}\right)=\frac{n p}{2 l}-\frac{n(q-1)}{2 l q}, \\
& \gamma^{\prime}=\frac{n p}{2 l}+p \cdot \frac{n}{2 l}\left(1-\frac{q-2}{p q}\right)=\frac{n p}{l}-\frac{n(q-2)}{2 l q} .
\end{aligned}
$$

It is clear from conditions (7), (8) and (29), (30) that

$$
\gamma^{\prime}>\gamma>1 \text {. }
$$

Allowing for (24), (25) we obtain that

$$
G_{1}(t)+G_{2}(t) \leq c, t \in\left[0, T_{\max }\right) .
$$

Thus the a priori estimate (9) is satisfied, so $T=\infty$. From (14) and (31) we yield the inequality (10).

\section{Nonexistence of Global Solutions}

Next let us discus the counterpart of the conditions (7) and (8). To this end we considered the Cauchy problem for the semi-linear hyperbolic inequalities

$$
\begin{aligned}
& u_{t t}+(-1)^{l} \Delta^{l} u+u_{t} \geq f(t, x, u), \\
& t>0, x \in R^{n}, \\
& u(0, x)=u_{0}(x), u_{t}(0, x)=u_{1}(x), \\
& x \in R^{n}
\end{aligned}
$$

where

$$
f(t, x, u)=\frac{1}{\left(1+|x|^{2}\right)^{s}}|u|^{p} .
$$

The weak solution of inequality (32) with initial data (33) where

$$
u_{0}(.) \in W_{1}^{l}\left(R^{n}\right), u_{1}(.) \in L_{1}\left(R^{n}\right)
$$

is called a function $u(t, x) \in L_{1}\left(R_{+} \times R^{n}\right)$ which, and $u(t, x)$ satisfies the following inequality:

$$
\begin{aligned}
& -\int_{R^{n}}\left[u_{0}(x)+u_{1}(x)\right] \zeta(0, x) \mathrm{d} x+\int_{R^{n}} u_{0}(x) \frac{\partial \zeta(0, x)}{\partial t} \mathrm{~d} x \\
& +\int_{0}^{\infty} \int_{R^{n}} u(t, x)\left[\zeta_{t t}(t, x)-\zeta_{t}(t, x)+(-1)^{l} \Delta^{l} \zeta(t, x)\right] \mathrm{d} x \mathrm{~d} t \\
& \geq \int_{0}^{\infty} \int_{R^{n}} f(t, x, u(t, x)) \zeta(t, x) \mathrm{d} x \mathrm{~d} t,
\end{aligned}
$$

for any function $\zeta(.) \in C_{0}^{2,2 l}\left(R_{+} \times R^{n}\right)$, where $\zeta(t, x) \geq 0,(t, x) \in R_{+} \times R^{n}$.

From Theorem 1 it follows that if $n \leq 2 l$ and

$$
p \in\left(1+\frac{2 l-2 s}{n},+\infty\right),
$$

then there exists $\delta_{0}>0$ such that for any $\left(u_{0}(),. u_{1}().\right) \in U_{\delta_{0}}$, problems (30) and (31) have a unique solution

$$
\begin{array}{r}
u(t, x) \in C\left([0, \infty) ; W_{2}^{l}\left(R^{n}\right)\right) \\
\cap C^{1}\left([0, \infty) ; L_{2}\left(R^{n}\right)\right)
\end{array} .
$$

Theorem 2. Let

$$
1<p \leq 1+\frac{2 l-2 s}{n},
$$

and

$$
\int_{R^{3}}\left[u_{0}(x)+u_{1}(x)\right] \mathrm{d} x \geq 0 .
$$

Then problems (32) and (33) have no nontrivial solutions.

\section{Proof of Theorem 2}

We assume that $u(t, x)$ is a global solution of (32) and (33). Let $\phi \in C^{2}(R ;[0,1])$ be such that

$$
\phi(r)=1, r \leq 1, \phi(r)=0, r \geq 2
$$

and, choose

$$
\zeta(t, x)=\phi\left(\frac{t^{2}+|x|^{2 l}}{R^{2 l}}\right), R>0 \quad \text { (see [8]). }
$$

Taking such a $\zeta(t, x)$ as the test function in Definition 1 , we get that 


$$
\begin{aligned}
& \int_{R^{n}}\left[u_{0}(x)+u_{1}(x)\right] \zeta(0, x) \mathrm{d} x \\
& +\int_{0}^{\infty} \int_{R^{n}} \frac{1}{\left(1+|x|^{2}\right)^{s}}|u(t, x)|^{p} \zeta(t, x) \mathrm{d} x \mathrm{~d} t \\
& \leq \int_{R^{n}} u_{0}(x) \frac{\partial \zeta(0, x)}{\partial t} \mathrm{~d} x \\
& +\int_{0}^{\infty} \int_{R^{n}} u(t, x)\left[\zeta_{t t}(t, x)-\zeta_{t}(t, x)\right. \\
& \left.+(-1)^{l} \Delta^{l} \zeta(t, x)\right] \mathrm{d} x \mathrm{~d} t .
\end{aligned}
$$

The choose of $\zeta($.$) implies that$

$$
\int_{R^{n}} u_{0}(x) \frac{\partial \zeta(0, x)}{\partial t} \mathrm{~d} x=0 .
$$

Define $\Omega=\left\{(t, x) \in[0, \infty) \times R^{n}, t^{2}+|x|^{2 l} \leq 2\right\}$. Again, by the choice of $\zeta(t, x)$, it is easy to show that

$$
\begin{aligned}
& \int_{0}^{\infty} \int_{R^{n}}\left(1+|x|^{2}\right)^{\frac{s p^{\prime}}{p}} \zeta^{-\frac{p^{\prime}}{p}}\left|\zeta_{t}\right|^{p^{\prime}} \mathrm{d} x \mathrm{~d} t \leq C_{1}<\infty, \\
& \int_{0}^{\infty} \int_{R^{n}}\left(1+|x|^{2}\right)^{\frac{s p^{\prime}}{p}} \zeta^{-\frac{p^{\prime}}{p}}\left|\zeta_{t t}\right|^{p^{\prime}} \mathrm{d} x \mathrm{~d} t \leq C_{2}<\infty, \\
& \int_{0}^{\infty} \int\left(1+|x|^{2}\right)^{\frac{s p^{\prime}}{p}} \zeta^{-\frac{p^{\prime}}{p}}\left|\Delta^{l} \zeta\right|^{p^{\prime}} \mathrm{d} x \mathrm{~d} t \leq C_{3}<\infty,
\end{aligned}
$$

Take scaled variables $t=\lambda^{2 l} \tau, x_{i}=\lambda y_{i}, i=1, \cdots, n$, then we have

$$
\begin{aligned}
& \int_{R^{n}}\left[u_{0}(x)+u_{1}(x)\right] \zeta(0, x) \mathrm{d} x \\
& +\int_{0}^{\infty} \int_{R^{n}} \frac{1}{\left(1+|x|^{2}\right)^{s}}|u(t, x)|^{p} \zeta(t, x) \mathrm{d} x \mathrm{~d} t \\
& \leq \lambda^{\sigma_{1}} \eta_{1}+\lambda^{\sigma_{2}} \eta_{2}+\lambda^{\sigma_{3}} \eta_{3},
\end{aligned}
$$

where

$$
\begin{aligned}
& \eta_{1}= \\
& c_{2} \iint_{\Omega}\left(\frac{1}{\lambda^{2 / l \mu}}+|y|^{2}\right)^{\frac{s p^{\prime}}{p}}(\phi \circ \rho)^{-\frac{p^{\prime}}{p}}\left|(\phi \circ \rho)_{\tau \tau}\right|^{p^{\prime}} \mathrm{d} y \mathrm{~d} \tau \leq c, \\
& \eta_{2}= \\
& c_{2} \iint_{\Omega}\left(\frac{1}{\lambda^{2 / l \mu}}+|y|^{2}\right)^{\frac{s p^{\prime}}{p}}(\phi \circ \rho)^{-\frac{p^{\prime}}{p}}\left|(\phi \circ \rho)_{\tau}\right|^{p^{\prime}} \mathrm{d} y \mathrm{~d} \tau \leq c, \\
& \eta_{3}= \\
& c_{3} \iint_{\Omega}\left(\frac{1}{\lambda^{2 / l \mu}}+|y|^{2}\right)^{\frac{s p^{\prime}}{p}}(\phi \circ \rho)^{-\frac{p^{\prime}}{p}}\left|\Delta^{l}(\phi \circ \rho)\right|^{p^{\prime}} \mathrm{d} y \mathrm{~d} \tau \leq c,
\end{aligned}
$$

$$
\begin{aligned}
& \sigma_{1}=\frac{2 s}{p-1}-\frac{4 l p}{p-1}+2 l+n, \\
& \sigma_{2}=\sigma_{3}=\frac{2 s}{p-1}-\frac{2 l p}{p-1}+n .
\end{aligned}
$$

Letting $\lambda \rightarrow \infty$ in (39), owing to (35), (40), (41) we get

$$
\begin{aligned}
& \int_{R^{n}}\left[u_{0}(x)+u_{1}(x)\right] \mathrm{d} x \\
& +\int_{0}^{\infty} \int_{R^{n}} \frac{1}{\left(1+|x|^{2}\right)^{s}}|u(t, x)|^{p} \mathrm{~d} x \mathrm{~d} t \leq C<\infty .
\end{aligned}
$$

Taking into account condition (36), from (45) it follows that

$$
\int_{0}^{\infty} \int_{R^{n}} \frac{1}{\left(1+|x|^{2}\right)^{s}}|u(t, x)|^{p} \mathrm{~d} x \mathrm{~d} t \leq C<\infty .
$$

Further, by applying the Holder inequality, from (37) we obtain

$$
\begin{aligned}
& \int_{|x|<\lambda}\left[u_{0}(x)+u_{1}(x)\right] h\left(\frac{|x|^{2 l}}{\lambda^{4}}\right) \mathrm{d} x \\
& +\int_{0}^{\infty} \int_{R^{n}} \frac{1}{\left(1+|x|^{2}\right)^{s}}|u(t, x)|^{p} \varsigma(t, x) \mathrm{d} x \mathrm{~d} t \\
& \leq\left(\iint_{\lambda^{2} \leq t^{2}+|x|^{4} \leq 2 \lambda^{2}} \frac{1}{\left(1+|x|^{2}\right)^{s}}|u(t, x)|^{p} \mathrm{~d} x \mathrm{~d} t\right)^{1 / p} \\
& \times\left(\iint_{\lambda^{2} \leq t^{2}+\left.x\right|^{4} \leq 2 \lambda^{2}} \frac{1}{\left(1+|x|^{2}\right)^{s p^{\prime}}}\left|\zeta_{t t}+\zeta_{t}+\Delta^{l} \zeta\right|^{p^{\prime}} \mathrm{d} x \mathrm{~d} t\right) .
\end{aligned}
$$

Letting $\lambda \rightarrow \infty$ in (47), owing to (45), we get

$$
\int_{R^{n}}\left[u_{0}(x)+u_{1}(x)\right] \mathrm{d} x+\int_{0}^{\infty} \int_{R^{n}} \frac{1}{\left(1+|x|^{2}\right)^{s}}|u(t, x)|^{p} \mathrm{~d} x \mathrm{~d} t \leq 0 .
$$

Finally, taking into condition (36), we have that

$$
u(t, x)=0 \text {. }
$$

\section{Acknowledgments}

This work was supported by the Science Development Foundation under the President of the Republic of Azerbaijan Grant No EIF-2011-1(3)-82/18-1.

\section{REFERENCES}

[1] A. B. Aliev and A. A. Kazymov, "Global Weak Solutions 
of the Cauchy Problem for Semi-Linear Pseudo-Hyperbolic Equations,” Differential Equations, Vol. 45, No. 2, 2009, pp. 1-11.

[2] A. B. Aliev and B. H. Lichaei, "Existence and Non-Existence of Global Solutions of the Cauchy Problem for Higher Semi-Linear Pseudo-Hyperbolic Equations,” Nonlinear Analysis, Theory, Methods and Applications, Vol. 72, No. 7-8, 2010, pp. 3275-3288.

[3] B. R. Ikehata, Y. Miataka and Y. Nakatake, "Decay Estimates of Solutions of Dissipative Wave Equations in $R_{n}$ with Lower Power Nonlinearities," Journal of the Mathematical Society of Japan, Vol. 56, No. 2, 2004, pp. 365373.

[4] T. Li and Y. Zhou, "Breakdown of Solutions $u+u_{t}=$ $|u|^{1+\alpha}, "$ Discrete and Continuous Dynamical Systems, Vol. 1, No. 4, 1995, pp. 503-520. doi:10.3934/dcds.1995.1.503

[5] I. E. Segal, "Dispersion for Non-Linear Realistic Equations II," Journal of the American Mathematical Society, Vol. 4, No. 16, 1968, pp. 459-497.

[6] Q. S. Zhag, “A Blow-Up Result for a Nonlinear Wave
Equation with Damping," The Critical Case, dans Comptesrendus de l'Académie des Sciences de Paris, Serie I, Vol. 333, 2001, pp. 109-114.

[7] G. Todorova and B. Yordanov, "Critical Exponent for a Nonlinear Wave Equation with Damping," dans Comptes rendus de l'Académie des Sciences de Paris, Serie I, Vol. 330, 2000, pp. 557-562.

[8] E. Mitidieri and S. I. Pokhozhaev, “A Priori Estimates and the Absence of Solutions of Nonlinear Partial Differential Equations and Inequalities," Proceedings of the Steklov Institute of Mathematics, Vol. 234, 2001, pp. 1-362.

[9] A. B. Aliev, "Solvability in the Large of the Cauchy Problem for Quasilinear Equations of Hyperbolic Type," Doklady Akademii Nauk SSSR, Vol. 240, No. 2, 1978, pp. 249- 252.

[10] O. V. Besov, V. P. Ilin and S. M. Nikolski, "Integral Representation of Functions and Embedding Theorem," V.H. Wilson and Sons, Washington DC, 1978. 